\title{
Diffusion in stationary flow from mesoscopic nonequilibrium thermodynamics
}

\author{
I. Santamaría-Holek, D. Reguera, and J. M. Rubí \\ Departament de Física Fonamental-CER Física de Sistemes Complexos, Facultat de Física, Universitat de Barcelona, Diagonal 647, \\ 08028 Barcelona, Spain \\ (Received 12 December 2000; published 18 April 2001)
}

\begin{abstract}
We analyze the diffusion of a Brownian particle in a fluid under stationary flow. By using the scheme of nonequilibrium thermodynamics in phase space, we obtain the Fokker-Planck equation that is compared with others derived from the kinetic theory and projector operator techniques. This equation exhibits violation of the fluctuation-dissipation theorem. By implementing the hydrodynamic regime described by the first moments of the nonequilibrium distribution, we find relaxation equations for the diffusion current and pressure tensor, allowing us to arrive at a complete description of the system in the inertial and diffusion regimes. The simplicity and generality of the method we propose makes it applicable to more complex situations, often encountered in problems of soft-condensed matter, in which not only one but more degrees of freedom are coupled to a nonequilibrium bath.
\end{abstract}

DOI: 10.1103/PhysRevE.63.051106

PACS number(s): 05.40.Jc, 05.70.Ln, 82.70.-y

\section{INTRODUCTION}

Liquid matter when subjected to the action of external forces or gradients exhibits peculiar characteristics that do not manifest in the absence of those external inputs. Its statistical mechanical properties present significant features as the appearance of long-range correlations and phase transitions, or the violation of the fluctuation-dissipation theorem (see, for example, Refs. [1-7]). The aim of nonequilibrium statistical mechanical theories is precisely to explain the behavior of systems in such situations, away from equilibrium.

These characteristics, far from being specific of pure substances as simple fluids [5], also manifest in complex fluids [8]. Typical situations of transport in liquids or liquidlike systems involve the joint motion of liquid and solid or solidlike phases under the action of an external input. Many examples can be found in systems as polymers [9], suspensions of neutral [10] and field-responsive particles [11], and granular media under shear flow [12]. The presence of shear flow significantly modifies transport properties and may induce the appearance of phases that otherwise would remain hidden (see, for instance, Refs. [13-15]). This is the reason why the influence of the shear flow in the dynamics is a subject that has received much attention in the last years, specially in the domain of soft-condensed matter.

Our purpose in this paper is precisely to analyze one of the simplest examples of a system coupled to a nonequilibrium bath, whose physical realization is a set of noninteracting Brownian particles moving in a fluid in stationary flow. The suspended objects diffuse in and are convected by the fluid; their motion may eventually be influenced by the presence of external fields. These are the basic ingredients controlling the dynamics of the suspended phase. Since the Brownian objects are of mesoscopic nature, the dynamic description demands a mesoscopic treatment in terms of a probability distribution function. The evolution in time of this quantity is governed by Fokker-Planck and Smoluchowski equations. These equations constitute the basis for a mesoscopic description of the system and enable one to extract macroscopic information from the evolution equations for the moments of the distribution. It becomes then of primary importance to establish simple methods able to provide expressions of those equations in situations outside equilibrium.

Kinetic equations of the Fokker-Planck type have been basically derived from kinetic theory of gases using the diffusion approximation in the Boltzmann equation $[1,16,17]$, from the theory of stochastic processes through the master equation [18] or by means of projection operator techniques $[19,20]$. It has also been shown that these kinetic equations can be derived from mesoscopic nonequilibrium thermodynamics (MNET) [21-28]. As in nonequilibrium thermodynamics, the basic point of this theory consists of assuming local equilibrium, which is performed at a more basic level: at mesoscopic level. This fact enables one to formulate a Gibbs equation in which the entropy, in accordance with the concept of Gibbs entropy, also depends on a "density": the probability density. By applying the rules of nonequilibrium thermodynamics, one obtains the entropy production and from it the corresponding linear laws between fluxes and forces. When using these laws in the continuity (balance) equation for the probability density one obtains the FokkerPlanck equation $[21,22]$. This is precisely the scheme we will adopt in this paper to analyze diffusion in stationary flow in both, the kinetic and the hydrodynamic regimes.

The paper is organized as follows. In Sec. II, we derive the Fokker-Planck equation describing Brownian motion under the presence of an arbitrary steady flow, in the framework of MNET. In Sec. III, we discuss the hydrodynamic description by constructing the hierarchy of moments of the distribution function accounting for the macroscopic evolution of the system. These equations reveal the presence of inertial and diffusion regimes for the dynamics of the particles. In Sec. IV, we analyze the particular case of a shear flow. The corresponding Fokker-Planck equation is compared with the one obtained in [29] by means of kinetic theory. In the diffusion regime, we give explicit expressions for the pressure tensor and the viscosity. Finally, in the discussion, we summarize our main results and indicate potential applications of the formalism we have established to soft-condensed matter systems. 


\section{FOKKER-PLANCK DYNAMICS FROM MESOSCOPIC NONEQUILIBRIUM THERMODYNAMICS}

We consider a dilute suspension of spherical particles of mass $m$ immersed in a liquid phase with constant density that acts as a heat bath. The whole system is subjected to conditions creating a stationary flow described by the velocity field

$$
\vec{v}=\vec{v}_{0}(\vec{r})
$$

Since our main purpose is to analyze the effect of the velocity gradient on the dynamics of the particle, we will consider isothermal conditions neglecting "viscous heating."

The mesoscopic nature of the suspended phase makes it necessary to analyze its dynamics by means of a FokkerPlanck equation accounting for the evolution of the distribution function, which may, in general, depend on the coordinates and momenta necessary to specify the state of the suspended objects. Since we assume no direct interactions between particles, the Brownian "gas" will be described by means of the single-particle distribution function $f(\vec{r}, \vec{u}, t)$, which depends explicitly on the position $\vec{r}$, particle velocity $\vec{u}$, and time $t$.

The first step towards the obtention of the Fokker-Planck equation is the formulation of the conservation laws for the gas of suspended particles. In the absence of external body forces, the distribution function obeys the continuity equation

$$
\frac{\partial f}{\partial t}+\vec{\nabla} \cdot \vec{u} f=-\frac{\partial}{\partial \vec{u}} \cdot \vec{J} \vec{u},
$$

which introduces the current $\vec{J}_{\vec{u}}$ in phase space. The average of Eq. (2) with respect to the particle velocity $\vec{u}$ leads to the macroscopic equation for the balance of mass, which can be written in the form

$$
\frac{d \rho}{d t}=-\rho \vec{\nabla} \cdot \vec{v}
$$

Here $\rho(\vec{r}, t)$ is the density of the Brownian gas, given by

$$
\rho(\vec{r}, t)=m \int f d \vec{u},
$$

$\vec{v}(\vec{r}, t)$ is the average velocity of the Brownian particles defined through the expression

$$
\rho \vec{v}(\vec{r}, t)=m \int \vec{u} f d \vec{u} .
$$

and we have defined the total derivative as

$$
\frac{d}{d t} \equiv \frac{\partial}{\partial t}+\vec{v} \cdot \vec{\nabla}
$$

Our purpose is to obtain the equation governing the evolution of the probability density $f$, therefore we need to find out the explicit expression for the current $\vec{J}_{\vec{u}}$. To this end, we will assume local equilibrium for which entropy variations are given through the Gibbs equation $[21,22]$

$$
\delta s=\frac{1}{T} \delta e+\frac{1}{T} p \delta \rho^{-1}-m \int \frac{\mu}{T} \delta c_{\vec{u}} d \vec{u} .
$$

Here $s(\vec{r}, t)$ and $e(\vec{r}, t)$ are the entropy and total energy per unit mass of the Brownian particles, respectively, $p(\vec{r}, t)$ is the hydrostatic pressure, $\mu(\vec{u}, \vec{r}, t)$ is the nonequilibrium chemical potential per unit mass, and $c_{\vec{u}}=m f / \rho$ is the Brownian mass fraction [21]. Notice that the term including the chemical potential in Eq. (7) is reminiscent of the corresponding one for a mixture in which the different components would be specified by the continuum "index" $\vec{u}$. Following the scheme of nonequilibrium thermodynamics [21], we will assume that Eq. (7) remains valid for changes in time and position into a mass element followed along the center of gravity motion of the Brownian gas.

Since the particles undergo only translational motion, and in view of Eqs. (2), (3), and (7), the remaining conservation law is the one for the energy. The presence of the external flow is responsible for the appearance in that equation of the term of "viscous heating," giving rise to variations in the temperature field. To maintain isothermal conditions, and following previous ideas introduced in the implementation of the so-called "homogeneous shear" [30,31], we will assume the existence of a local heat source capable to remove the heat generated in the process. Under this assumption, the energy of the volume elements remains constant along their paths and its balance equation can be omitted in the subsequent analysis.

The expression of the nonequilibrium chemical potential can be found through the Gibbs entropy postulate for the Brownian particles, written as

$$
s=-k_{B} \int c_{\vec{u}} \ln \frac{f}{f^{l \cdot e q}} d \vec{u}+s^{l . e q} .
$$

Here $k_{B}$ is Boltzmann's constant and $f^{l . e q}$ is the local equilibrium distribution function corresponding to the reference state described by the local Maxwellian with respect to the stationary convective flow [1],

$$
f^{l \cdot e q}(\vec{u}, \vec{r}, t)=e^{[m / k T]\left[\mu_{B}-(1 / 2)\left(\vec{u}-\vec{v}_{0}\right)^{2}\right]} .
$$

In this expression $\mu_{B}(\vec{r}, t)$ is the local equilibrium chemical potential per unit mass of the Brownian particles. The local equilibrium entropy for the Brownian particles per unit mass is given by

$$
s^{l . e q}=-\frac{m}{T} \int c_{\vec{u}} \mu_{B} d \vec{u}+\frac{1}{T} e .
$$

After applying the total derivative in Eqs. (7), (8), and (10), and comparing the corresponding expressions for the 
time variations of the entropy, we arrive at the desired expression for the nonequilibrium chemical potential

$$
\mu=\frac{k_{B} T}{m} \ln f+\frac{1}{2}\left(\vec{u}-\vec{v}_{0}\right)^{2} .
$$

This expression, together with the equation for the Brownian mass fraction

$$
\frac{d}{d t} C_{\vec{u}}=-\frac{1}{\rho} \vec{\nabla} \cdot\left[\left(\vec{u}-\vec{v}_{0}\right) f\right]-\frac{\partial}{\partial \vec{u}} \cdot \vec{J}_{\vec{u}},
$$

obtained by combining Eq. (1), the continuity equation (2), and the balance of mass (3), is now substituted into Eq. (7). The resulting expression is integrated by parts over the velocity space assuming that the fluxes vanish at the boundaries. One then obtains the entropy balance equation in the form

$$
\rho \frac{d s}{d t}=-\vec{\nabla} \cdot \vec{J}_{s}+\sigma,
$$

where the entropy flux is given by

$$
\begin{aligned}
\vec{J}_{s}= & -k_{B} \int\left(\vec{u}-\vec{v}_{0}\right) f(\ln f-1) d \vec{u} \\
& -\frac{m}{2 T} \int\left(\vec{u}-\vec{v}_{0}\right) f\left(\vec{u}-\vec{v}_{0}\right)^{2} d \vec{u}
\end{aligned}
$$

and the entropy production is

$$
\sigma=-\frac{m}{T} \int \vec{J}_{u} \cdot \frac{\partial \mu}{\partial \vec{u}} d \vec{u}-\frac{m}{T} \int \vec{J} \cdot\left(\vec{u}-\vec{v}_{0}\right) \cdot \vec{\nabla} \vec{v}_{0} d \vec{u}
$$

This quantity consists of two contributions of the type fluxforce pair: the first one arises from the diffusion process in $\vec{u}$ space whereas the second is due to the presence of the convective flow $\vec{J} \equiv\left(\vec{u}-\vec{v}_{0}\right) f$. Since both the contributions are vectors, the fluxes couple to the two forces giving rise to cross effects. Following the nonequilibrium thermodynamics rules, we can establish linear phenomenological relationships between fluxes and thermodynamic forces. Assuming locality in $\vec{u}$ space, for which only fluxes and forces with the same value of $\vec{u}$ are coupled, the expressions for the currents are the following:

$$
\begin{gathered}
\vec{J}_{\vec{u}}=-\frac{m}{T} \overrightarrow{\vec{L}}_{\vec{u} \vec{u}} \cdot \frac{\partial \mu}{\partial \vec{u}}-\frac{m}{T} \overrightarrow{\vec{L}}_{\vec{u} r} \cdot\left(\vec{u}-\vec{v}_{0}\right) \cdot \vec{\nabla} \vec{v}_{0}, \\
\vec{J}=-\frac{m}{T} \vec{L}_{\vec{r} r} \cdot\left(\vec{u}-\vec{v}_{0}\right) \cdot \vec{\nabla} \vec{v}_{0}-\frac{m}{T} \vec{L}_{\vec{r} \vec{u}} \cdot \frac{\partial \mu}{\partial \vec{u}},
\end{gathered}
$$

where $\overrightarrow{\vec{L}}_{\vec{u} u}, \overrightarrow{\vec{L}}_{\vec{u} r}, \overrightarrow{\vec{L}}_{\vec{r} u}$, and $\overrightarrow{\vec{L}}_{\vec{r} \vec{r}}$ are Onsager coefficients. These coefficients may, in general, depend on the imposed velocity gradient. In this case they satisfy generalized Onsager relations [32] in which time-reversal symmetry must also be applied to the external driving. In the case we are considering, we have $\overrightarrow{\vec{L}}_{\vec{u}}=-\overrightarrow{\vec{L}}_{\vec{r} u}$. Defining now the tensors

$$
\vec{\alpha}=\frac{m \overrightarrow{\vec{L}}_{\vec{u} u}}{f T}, \quad \overrightarrow{\vec{\xi}}=\frac{m \overrightarrow{\vec{L}}_{\vec{r} r}}{f T}, \quad \vec{\epsilon}=\frac{m \overrightarrow{\vec{L}}_{\vec{u}}}{f T}
$$

and using the expression for the chemical potential (11), the fluxes can be recast in the form

$$
\begin{gathered}
\vec{J}_{\vec{u}}=-\left(\vec{u}-\vec{v}_{0}\right) \cdot\left[\vec{\alpha}+\vec{\nabla} \vec{v}_{0} \cdot \overrightarrow{\vec{\epsilon}}\right] f-\frac{k_{B} T}{m} \vec{\alpha} \cdot \frac{\partial f}{\partial \vec{u}}, \\
\vec{J}=-\left(\vec{u}-\vec{v}_{0}\right) \cdot\left[\nabla \vec{v}_{0} \cdot \vec{\xi}-\overrightarrow{\vec{\epsilon}}\right] f+\frac{k_{B} T}{m} \overrightarrow{\vec{\epsilon}} \cdot \frac{\partial f}{\partial \vec{u}} .
\end{gathered}
$$

By substituting Eq. (19) into the continuity equation (2) for the single-particle distribution function, we finally obtain

$$
\frac{\partial f}{\partial t}+\vec{\nabla} \cdot \vec{u} f=\frac{\partial}{\partial \vec{u}} \cdot\left\{\left(\vec{u}-\vec{v}_{0}\right) \cdot[\vec{\alpha}+\nabla \vec{v} \cdot \overrightarrow{\vec{\epsilon}}] f+\frac{k_{B} T}{m} \vec{\alpha} \cdot \frac{\partial f}{\partial \vec{u}}\right\},
$$

which constitutes the Fokker-Planck equation describing the evolution of the nonequilibrium single-particle distribution function. The fact that the coefficients appearing in the equation are tensors reflects the anisotropy of the system induced by the imposed external flow. Moreover, this equation exhibits the fact that the flow breaks the Einstein relation by adding a term that depends on the imposed velocity gradient. This breaking constitutes a proof that the fluctuationdissipation theorem cannot be applied when fluctuations take place around the steady state.

The Fokker-Planck equation we have obtained can be compared with the ones derived by means of different methods. In Ref. [29], authors found a similar Fokker-Planck equation for the particular case of a shear flow. In Sec. IV, we will discuss this similarity in more details. Following time-dependent projector-operator techniques, a FokkerPlanck equation similar to ours was derived in [33] for the translational modes of a Brownian particles moving in a flowing bath under a temperature gradient. Since diffusion in a bath under temperature gradient was studied previously in [22] in the framework of MNET, the relevant point to emphasize here is the fact that the term including the velocity gradient enters the Fokker-Planck equation as an external force, in a similar way as in the equation obtained in [33].

\section{THE HYDRODYNAMIC EQUATIONS}

In the absence of direct interactions, the single-particle distribution function provides the complete description of the system at mesoscopic level. Macroscopically, the description must be carried out in terms of the moments of the distribution function, which are related to the hydrodynamic fields: density, momentum, and pressure tensor.

The density defined through Eq. (4) corresponds to the zero-order moment. The first-order moment has been defined in Eq. (5). The second moment centered about the average 
velocity $\vec{v}$ of the Brownian gas corresponds to the pressure tensor

$$
\vec{P}=m \int(\vec{u}-\vec{v})(\vec{u}-\vec{v}) f d \vec{u}
$$

Finally, the third centered moment, which is related to the flux of kinetic energy and stress, is given by

$$
\overrightarrow{\hat{Q}}=m \int(\vec{u}-\vec{v})(\vec{u}-\vec{v})(\vec{u}-\vec{v}) f d \vec{u}
$$

The set of evolution equations for these moments can be obtained by using the Fokker-Planck equation (21) in the definitions of the conserved quantities after performing the time derivative and the required integrations in velocity space [25]. The evolution equations for the three first moments are, respectively, the continuity equation

$$
\frac{\partial \rho}{\partial t}=-\vec{\nabla} \cdot \rho \vec{v}
$$

the balance of momentum

$$
\rho \frac{d \vec{v}}{d t}+\vec{\nabla} \cdot \overrightarrow{\vec{P}}=-\rho\left(\vec{v}-\vec{v}_{0}\right) \cdot \overrightarrow{\vec{C}}
$$

and the equation for the evolution in time of the pressure tensor

$$
\frac{d}{d t} \overrightarrow{\vec{P}}+2(\overrightarrow{\vec{P}} \cdot \vec{\nabla} \vec{v})^{s}+\overrightarrow{\vec{P}} \vec{\nabla} \cdot \vec{v}+2(\overrightarrow{\vec{P}} \cdot \overrightarrow{\vec{C}})^{s}=\frac{2 k_{B} T}{m} \rho \overrightarrow{\alpha^{s}}-\vec{\nabla} \cdot \overrightarrow{\vec{Q}},
$$

where $\overrightarrow{\vec{C}}=\left[\overrightarrow{\vec{\alpha}}+\vec{\nabla} \vec{v}_{0} \cdot \overrightarrow{\vec{\epsilon}}\right]$ and an upper $s$ means symmetric part of a tensor. In a similar way, we could derive the evolution equations for the higher-order moments of the distribution, which constitute a coupled hierarchy of hydrodynamic equations [25,34].

Notice that in Eq. (25), the right-hand-side term can be identified with the hydrodynamic force exerted by the fluid on the particle with $\overrightarrow{\vec{C}}$ playing the role of the friction constant. That friction constant establishes the characteristic relaxation time scale for the velocity and it is usually very large. For instance, for a mesoscopic particle of radius $a$ $\sim 10^{-5} \mathrm{~cm}$, moving in a quiescent liquid of viscosity $\eta$ $\sim 10^{-2} P$, the Stokes formula can be applied giving for the friction constant $\beta=6 \pi \eta a / m \sim 10^{8} \mathrm{~s}^{-1}$. Consequently, the discussion of the behavior of the system may be carried out by expanding the hierarchy of evolution equations for the moments in powers of $\overrightarrow{\vec{C}}^{-1}$. From the hierarchy of moments, one can easily realize that the ith moment introduces corrections that are of order $\overrightarrow{\vec{C}}^{-(i-1)}$ for even moments and $\overrightarrow{\vec{C}}^{-i}$ for the odd ones. If we drop out the terms arising from the third and higher moments, we are neglecting corrections to the diffusion equation of order $\overrightarrow{\vec{C}}^{-3}$. Notice that in order to retain $\overrightarrow{\vec{C}}^{-3}$ corrections, one has to include not only the third- order moment equation but also the equation for the fourth moment, which is of the same order.

The set of equations (24)-(26) then govern the hydrodynamic behavior of the Brownian gas immersed in a fluid moving with velocity profile $\vec{v}_{0}$. The elements of the tensor $\vec{C}^{-1}$ constitute characteristic time scales whose existence motivates the separation of the dynamics into two welldifferentiated regimes: an inertial regime for $t \ll\left(C^{-1}\right)_{i j}$, characterized for the relaxation of the variables towards the diffusion regime, which is achieved for $t \gg\left(C^{-1}\right)_{i j}$. Both the regimes will be discussed in the following subsections.

\section{A. Inertial regime}

In order to discuss the inertial regime, it is convenient to rewrite Eq. (26) for the evolution of the pressure tensor in the following way:

$$
\frac{1}{2} \frac{d}{d t} \overrightarrow{\vec{P}}+\left(\overrightarrow{\vec{P}} \cdot \overrightarrow{\vec{\tau}}_{1}^{-1}\right)^{s}=\frac{k_{B} T}{m} \rho \overrightarrow{\vec{\alpha}^{s}},
$$

where we have defined the matrix of relaxation times

$$
\overrightarrow{\vec{\tau}}_{1}=\left[\overrightarrow{\vec{C}}+\vec{\nabla} \vec{v}+\frac{1}{2}(\vec{\nabla} \cdot \vec{v}) \overrightarrow{1}\right]^{-1}
$$

and we have neglected corrections to the diffusion equation of orders $\overrightarrow{\vec{C}}^{-3}$ and higher. In an analogous way, from the evolution equation for the momentum, Eq. (25), we obtain

$$
\frac{d \vec{J}_{D}}{d t}+\vec{J}_{D} \cdot \vec{\tau}_{2}^{-1}=\rho \overrightarrow{v_{0}} \cdot \overrightarrow{\vec{C}}-\vec{\nabla} \cdot \overrightarrow{\vec{P}}
$$

where $\vec{J}_{D} \equiv \rho \vec{v}$ and

$$
\overrightarrow{\vec{\tau}}_{2}=[\vec{C}+\overrightarrow{\hat{1}}(\vec{\nabla} \cdot \vec{v})]^{-1}
$$

is the corresponding matrix of relaxation times.

The previous equations describe the inertial regime in the dynamics of the Brownian particles subjected to stationary flow. This regime holds for times small enough compared to the characteristic relaxation times, identified with the components of the matrices $\overrightarrow{\vec{\tau}}_{1}$ and $\overrightarrow{\vec{\tau}}_{2}$.

Our description of the inertial regime is consistent with the generalized hydrodynamic description in which the diffusion coefficient depends on the wave vector [35]. This property has been shown in [25] for the case of a quiescent liquid.

\section{B. Diffusion regime}

For times larger than any characteristic relaxation time $t$ $\gg\left(C^{-1}\right)_{i j}$, the system enters the diffusion regime. In such a regime, the dynamics become well described by a Smoluchowski equation for the density distribution $\rho$ in the configuration space.

In order to find the Smoluchowski equation, we will first discuss the diffusion approximation in the evolution equations for the pressure tensor (27) and the momentum (28). Both equations involve inertial, friction, and velocity gradi- 
ent time scales. For $t \gg\left(C^{-1}\right)_{i j}$, time derivatives can be neglected when compared with terms proportional to $\stackrel{\vec{C}}{\mathrm{C}}$. Notice also that the term $\vec{\nabla} \cdot \vec{v}$ is essentially a time derivative, as follows from Eq. (3), and can accordingly be neglected. Taking this consideration into account, the equations for the pressure tensor and the momentum then reduce to

$$
(\overrightarrow{\vec{P}} \cdot \overrightarrow{\vec{C}})^{s}+(\overrightarrow{\vec{P}} \cdot \vec{\nabla} \vec{v})^{s}=\frac{k_{B} T}{m} \rho \overrightarrow{\alpha^{s}}
$$

and

$$
\vec{J}_{D}=\rho \vec{v}_{0}-(\vec{\nabla} \cdot \overrightarrow{\vec{P}}) \cdot \overrightarrow{\vec{C}}^{-1}
$$

respectively. The first equation represents a linear set of coupled algebraic equations for the components of the pressure tensor $\vec{P}$ in terms of the components of the tensors $\vec{C}$, $\nabla \vec{v}$, and $\vec{\alpha}$. Once the explicit expression for the pressure tensor has been found, it must be introduced into the momentum equation (30) yielding the constitutive equation for the diffusion current $\vec{J}_{D}$. The Smoluchowski equation is then obtained after substituting $\vec{J}_{D}$ into the balance of mass (24).

In the following section, we will apply the previous scheme to the particular case of a shear flow.

\section{DIFFUSION IN A SHEAR FLOW}

Inherent to nonequilibrium thermodynamics is the fact that it cannot provide explicit expressions for the phenomenological (transport) coefficients that must be borrowed from other theories. In our case, the still unspecified quantities are $\stackrel{\vec{\alpha}}{\alpha}$ and $\vec{\epsilon}$. When a specific velocity profile is given, expressions for those tensors can be obtained from the kinetic theory or hydrodynamics.

The formalism developed in the previous sections is valid for an arbitrary stationary velocity field. In this section we will focus our discussion on the particular case of Brownian motion in a shear flow. Our first task will be the identification of the phenomenological coefficients. As pointed out before, the second term on the right-hand side of Eq. (25) can be identified with the force per unit mass exerted on a suspended particle by the host fluid where $\overrightarrow{\vec{C}}$ plays the role of the friction tensor. In general, such a density force can be calculated from hydrodynamics, (see, for instance, Refs. $[36,37])$. For the shear flow case, the friction coefficient contains, in general, linear and quadratic contributions in $\zeta a$, where $\zeta$ is the inverse penetration length of the perturbations $\zeta=(\gamma / \nu)^{1 / 2}$, with $\gamma$ being the shear rate and $\nu$ the kinematic viscosity. For a Brownian particle under moderate shear flow, the term $\zeta a$ is very small, consequently the friction tensor can be approximated by $\vec{C} \simeq \beta \overrightarrow{1}$. This identification leads to the following expression for the tensor $\vec{\alpha}$ :

$$
\stackrel{\vec{\alpha}}{=}=\beta \overrightarrow{1}-\vec{\nabla} \vec{v}_{0} \cdot \overrightarrow{\vec{\epsilon}}
$$

By introducing the previous expressions into the FokkerPlanck equation (21), we finally obtain

$$
\frac{\partial f}{\partial t}+\vec{u} \cdot \vec{\nabla} f=\frac{\partial}{\partial \vec{u}} \cdot\left\{\beta\left(\vec{u}-\vec{v}_{0}\right) f+\frac{k_{B} T}{m} \vec{\alpha} \cdot \frac{\partial f}{\partial \vec{u}}\right\} .
$$

As concluded, also from Eq. (21), the particular form of Eq. (32) implies that the fluctuation-dissipation theorem is no longer valid when the fluid (heat bath) is sheared. Notice that the term that invalidates that theorem is proportional to the velocity gradient or to the inverse penetration length squared. The theorem remains applicable in the case in which the friction coefficient contains a correction proportional to $\zeta$ [38].

The Fokker-Planck equation (32) is similar to the corresponding one obtained in Ref. [29] from the kinetic theory. By expanding the collision operator in the mass ratio between the fluid and Brownian particles, authors derived a Fokker-Planck equation in which the diffusion tensor contains a correction to the Einstein formula proportional to the stress tensor of the fluid. Since this quantity is proportional to the velocity gradient, we conclude that the form of the diffusion tensor they find is similar to our expression (31), which allows the identification of the tensor $\overrightarrow{\vec{\epsilon}}$.

With the explicit form of the Fokker-Planck equation (32) in our mind, our purpose is now to discuss the macroscopic evolution of the system. Following the procedure indicated in Sec. III B, we find that for a shear flow, in the diffusion regime, the expression for the Brownian pressure tensor is

$$
\overrightarrow{\vec{P}}=\frac{k_{B} T}{m} \rho\left[\overrightarrow{\overrightarrow{1}}-\left\{\beta^{-1}(\overrightarrow{\overrightarrow{1}}+\overrightarrow{\vec{\epsilon}}) \cdot \nabla \vec{v}_{0}\right\}^{s}\right] .
$$

From this equation, we can conclude that Brownian motion of the particles contributes to the total pressure tensor of the suspension in two forms. The first contribution is the wellknown scalar kinetic pressure given by

$$
p=\frac{k_{B} T}{m} \rho,
$$

which is the equation of state for the ideal Brownian gas. The second contribution comes from the irreversible part $\overrightarrow{\Pi^{\prime}}$ of the Brownian pressure tensor, which can be written in the form

$$
\vec{\Pi}=-D_{0} \rho\left[(\overrightarrow{\overrightarrow{1}}+\stackrel{\vec{\epsilon}}{\boldsymbol{\Pi}}) \cdot \vec{\nabla} \vec{v}_{0}\right]^{0},
$$

where $D_{0}=k_{B} T / m \beta$ is the diffusion coefficient of a particle when the liquid is at rest and an upper 0 means symmetric traceless tensor. This last equation defines the Brownian viscosity tensor

$$
\stackrel{\vec{\eta}}{\eta}_{B}=D_{0} \rho(\overrightarrow{\overrightarrow{1}}+\overrightarrow{\vec{\epsilon}})
$$

which contains the "Brownian" viscosity $D_{0} \rho$ [39], and the contribution due to the coupling with the nonequilibrium bath, which is proportional to $\vec{\epsilon}$. Equations (35) and (36) account for the contributions to the irreversible part of the 
pressure tensor and shear viscosity coefficient of the suspension. No contribution to the bulk viscosity has been found since the shear flow is incompressible.

Following the steps described previously, we can obtain the diffusion current $\vec{J}_{D}$ in the limit $t \gg\left(C^{-1}\right)_{i j}$ in which Eq. (28) has the form

$$
\vec{J}_{D}=\rho \vec{v}_{0}-\beta^{-1} \vec{\nabla} \cdot \vec{P} .
$$

Introducing the expression for the pressure tensor (33) into the last equation, we obtain

$$
\vec{J}_{D}=\rho \vec{v}_{0}-\overrightarrow{\vec{D}} \cdot \vec{\nabla} \rho-\rho \vec{\nabla} \cdot \overrightarrow{\vec{D}}
$$

Here we have defined the diffusion tensor as

$$
\overrightarrow{\vec{D}}=D_{0}\left[\overrightarrow{\overrightarrow{1}}-\beta^{-1}\left\{(\overrightarrow{\overrightarrow{1}}+\overrightarrow{\vec{\epsilon}}) \cdot \vec{\nabla} \vec{v}_{0}\right\}^{0}\right] .
$$

An important consequence of this result is that the presence of a shear flow modifies the diffusion current with respect to the case of a quiescent liquid. Substituting Eq. (38) into the balance of mass, Eq. (24), we finally obtain the diffusion equation for the Brownian particle

$$
\frac{\partial \rho}{\partial t}=-\vec{\nabla} \cdot \rho \vec{v}_{0}+\vec{\nabla} \cdot(\overrightarrow{\vec{D}} \cdot \vec{\nabla} \rho)+\vec{\nabla} \cdot(\rho \vec{\nabla} \cdot \overrightarrow{\vec{D}}) .
$$

This equation is a generalization of the usual Smoluchowski equation in the sense that it contains the anisotropic diffusion coefficient (39) that depends on the imposed velocity gradient. For the particular case of shear flow, the velocity gradient is a constant, therefore $\overrightarrow{\vec{D}}$ does not exhibit spatial dependence and we recover the usual Smoluchowski equation

$$
\frac{\partial \rho}{\partial t}=-\vec{\nabla} \cdot \rho \vec{v}_{0}+\vec{\nabla} \cdot(\vec{D} \cdot \nabla \rho) .
$$

This equation coincides formally with the one found in Ref. [40]. The difference between both equations lies in the form of the diffusion tensor. In the cited work, author starts from a Fokker-Planck equation that satisfies the fluctuationdissipation theorem. In the diffusion regime the authors find, consistent with the approximation, that the diffusion tensor and the mobility of the Brownian particles in the fluid are related to each other through Einstein's formula.

\section{DISCUSSION}

In this paper we have analyzed the dynamics of the suspension of Brownian particles in a nonequilibrium situation resulting from the action of an externally imposed velocity gradient. We have applied the method of mesoscopic nonequilibrium thermodynamics to study the dissipation in phase space related to the underlying diffusion process of the probability density of the particles.

In MNET, local equilibrium is assumed at mesoscopic level. A Gibbs equation is then proposed in which the entropy depends on the probability density: the pertinent den- sity in phase space, according to the concept of Gibbs entropy. By applying the rules of nonequilibrium thermodynamics, one obtains the entropy production of the system, which enables one to derive the expression for the diffusion current in phase space and, consequently, to obtain the Fokker-Planck equation for the single-particle distribution function. This expression exhibits violation of the fluctuation-dissipation theorem whose origin is precisely the presence of the external gradient. This feature, commonly found in the wide class of driven-diffusion systems [4,38], has also been reported in slow relaxation process of glassy systems [41].

The hydrodynamic level of description is accomplished from the evolution equations for the first moments of the distribution function, which can be obtained through the Fokker-Planck equation. The time evolution of the moments include relaxation equations for the diffusion current and the pressure tensor, whose form permits to elucidate the existence of inertial (short-time) and diffusion (long-time) regimes. In the diffusion regime, the mesoscopic description is carried out by means of a Smoluchowski equation. In this regime, the equations for the moments coincide with the differential equations of nonequilibrium thermodynamics. The equations for the moments obtained from the Fokker-Planck equation in the framework of MNET extend the domain of applicability of nonequilibrium thermodynamics to shorter time scales. These equations can be reformulated in terms of transport coefficients that depend on the wave vector, in accordance with generalized hydrodynamics [35].

Our results can be compared with others obtained previously using different theories. The Fokker-Planck equation we have derived is similar to the one proposed in [29] for the case of a shear flow. As in our case, these authors show that when the system is sheared, the diffusion coefficient is no longer given by Stokes-Einstein law. It contains a correction proportional to the pressure tensor, which is basically of the same nature as the one we obtain, i.e., proportional to the velocity gradient. This fact clearly shows violation of the fluctuation-dissipation theorem due to the presence of the external input necessary to maintain the stationary state. Similar conclusions are obtained in [33], where the general case in which a stationary flow and a temperature gradient act simultaneously is studied by means of time-dependent projector-operator techniques.

Having discussed the comparison of our results with others coming from statistical mechanical theories, it remains to analyze them in the framework of thermodynamical theories dealing with systems outside equilibrium, in particular, with extended irreversible thermodynamics. This theory provides hydrodynamic equations that also contain relaxation terms for the pressure tensor and the diffusion current. Along our analysis of this and other cases of systems outside equilibrium (see Refs. $[24,25,27,42]$ ), we have shown that by simply using nonequilibrium thermodynamics or its extension to the mesoscopic domain (MNET), we are able to completely characterize the evolution of systems outside equilibrium. This fact questions the need of using generalized entropies [43] depending on nonthermodynamic variables: the fluxes, which constitute the cornerstone of extended irreversible 
thermodynamics. The application of the well-established nonequilibrium thermodynamics postulates as indicated in [21], suffices to provide a general scheme under which nonequilibrium processes of macroscopic and mesoscopic nature can be treated.

Far from being specific to the case of a suspension of noninteracting Brownian particles, we have treated in this paper, the method we have presented could systematically be applied to analyze the dynamics of soft-condensed matter systems under shear flow. For example, the case in which direct and hydrodynamic interactions among particles become important can be worked out along the lines indicated in [24] for the case of a quiescent liquid. If the suspended objects are deformable or need additional parameters to characterize their state, as may occur in polymers [9] or liquid crystals $[42,44]$, the set of variables of the distribution func- tion has to be enlarged. One would obtain the corresponding Fokker-Planck equation and from it the evolution equations for the moments that define the hydrodynamic or generalized hydrodynamic regimes. In all these cases, kinetic and hydrodynamic equations can be derived following the method of mesoscopic nonequilibrium thermodynamics.

\section{ACKNOWLEDGMENTS}

I.S.H. wants to acknowledge J. Dufty and A. Pérez Madrid for their comments, and UNAM-DGAPA for economic support. D.R. wishes to thank Generalitat de Cataluna for financial support. This work was partially supported by DGICYT of the Spanish Government under Grant No. PB981258.
[1] J.A. McLennan, Introduction to Non-equilibrium Statistical Mechanics (Prentice-Hall, Englewood Cliffs, NJ, 1989).

[2] J. Keizer, Statistical Thermodynamics of Nonequilibrium Processes (Springer-Verlag, New York, 1987).

[3] R. Schmitz, Phys. Rep. 171, 1 (1988).

[4] G.L. Eyink, J.L. Lebowitz, and H. Spohn, J. Stat. Phys. 83, 385 (1996).

[5] J.R. Dorfman, T.R. Kirkpatrick, and J.V. Sengers, Annu. Rev. Phys. Chem. 45, 213 (1994).

[6] I. Pagonabarraga and J.M. Rubí, Phys. Rev. E 49, 267 (1994).

[7] A. Onuki, J. Phys.: Condens. Matter 9, 6119 (1997).

[8] Soft and Fragile Matter, edited by M.E. Cates and M.R. Evans (Institute of Physics, Bristol, 2000).

[9] M. Doi and S.F. Edwards, The Theory of Polymer Dynamics (Oxford University Press, New York, 1988).

[10] T. G. M. van de Ven, Colloidal Hydrodynamics (Academic Press, London, 1989).

[11] J.M. Rubí and J.M.G. Vilar, J. Phys.: Condens. Matter 12, A75 (2000).

[12] H.M. Jaeger, S.R. Nagel, and R.P. Behringer, Rev. Mod. Phys. 68, 1259 (1996).

[13] Z.R. Chen et al., Science 277, 1248 (1997).

[14] C. Liu and D.J. Pine, Phys. Rev. Lett. 77, 2121 (1996).

[15] J. Yamamoto and H. Tanaka, Phys. Rev. Lett. 77, 4390 (1996).

[16] E.M. Lifshitz and L.P. Pitaevskii, Physical Kinetics (Pergamon Press, Oxford, 1981).

[17] D.N. Zubarev, Non-equilibrium Statistical Thermodynamics (Consultants Bureau, New York, 1974).

[18] N.G. van Kampen, Stochastic Processes in Physics and Chemistry (North-Holland, Amsterdam, 1992).

[19] N.G. van Kampen and I. Oppenheim, Physica A 138, 231 (1986).

[20] J.M. Deutch and I. Oppenheim, Faraday Discuss. Chem. Soc. 83, 1 (1987).
[21] S. R. de Groot and P. Mazur, Non-equilibrium Thermodynamics (Dover, New York, 1984).

[22] A. Pérez-Madrid, J.M. Rubí, and P. Mazur, Physica A 212, 231 (1994).

[23] I. Pagonabarraga, A. Pérez-Madrid, and J.M. Rubí, Physica A 237, 205 (1997).

[24] J.M. Rubí and P. Mazur, Physica A 250, 253 (1998).

[25] J.M. Rubí and A. Pérez-Madrid, Physica A 264, 492 (1999).

[26] P. Mazur, Physica A 261, 451 (1998).

[27] P. Mazur, Physica A 274, 491 (1999).

[28] J.M. Rubí and P. Mazur, Physica A 276, 477 (2000).

[29] R. Rodriguez, E. Salinas-Rodriguez, and J. Dufty, J. Stat. Phys. 32, 279 (1983).

[30] W.T. Ashurst and W.G. Hoover, Phys. Rev. A 11, 658 (1975).

[31] E. Peakock-Lopez and J. Keizer, Phys. Lett. 108A, 85 (1985).

[32] J.W. Dufty and J.M. Rubí, Phys. Rev. A 36, 222 (1987).

[33] J.E. Shea and I. Oppenheim, Physica A 250, 265 (1998).

[34] G. Wilemski, J. Stat. Phys. 14, 153 (1976).

[35] J. P. Boon and S. Yip, Molecular Hydrodynamics (Dover, New York, 1980).

[36] P.G. Saffman, J. Fluid Mech. 22, 385 (1965).

[37] A. Pérez-Madrid, J.M. Rubí, and D. Bedeaux, Physica A 163, 778 (1990).

[38] J.M. Rubí and D. Bedeaux, J. Stat. Phys. 53, 125 (1988).

[39] K.F. Freed and M. Muthukumar, J. Chem. Phys. 69, 2657 (1978).

[40] B.U. Felderhof, Physica A 147, 203 (1987).

[41] J.P. Bouchaud, L.F. Cugliandolo, J. Kurchan, and M. Mzard, in Spin Glasses and Random Fields, edited by A.P. Young (World Scientific, Singapore, 1997).

[42] D. Bedeaux and J.M. Rubí (unpublished).

[43] D. Jou, J. Casas-Vazquez, and G. Lebon, Rep. Prog. Phys. 62, 1035 (1999).

[44] P.G. de Gennes, The Physics of Liquid Crystals (Clarendon Press, Oxford, 1974). 